\title{
MILLENNIALS Y DESTINOS TURÍSTICOS: CASO TABASCO, MÉXICO
}

\section{MILLENNIALS AND TOURIST DESTINATIONS: THE CASE OF TABASCO, MEXICO}

\author{
Lucía Sandoval Núñez ${ }^{1 \star}$, María Lyssette Mazó Quevedo², Juan Eduardo Uribe Domínguez ${ }^{3}$ \\ 1,3 - Universidad Tecnológica de Tabasco, Tabasco, México \\ 2 - Universidad Popular de la Chontalpa, Tabasco, México
}

1. Email: luciasandoval1@hotmail.com ORCID: https://orcid.org/0000-0003-0803-6035

2. Email: marialyssette@gmail.com ORCID: http://orcid.org/0000-0001-9174-3704

3. Email: eduribe2496@gmail.com ORCID: https://orcid.org/0000-0002-2563-7644

Recibido: 24/11/2021 Aceptado: 13/01/2022

Para Citar: Sandoval Núñez, L., Mazo Quevedo, M. L., \& Uribe Domínguez, J. E. (2022). Millennials y destinos turísticos: caso Tabasco, México. Revista Publicando, 9(33), 48-66. https://doi.org/10.51528/rp.vol9.id2280

\section{Resumen: \\ El objetivo del artículo es identificar los factores que influyen en la elección de un destino turístico en la generación conocida como millennials. El enfoque metodológico presentado es cuantitativo, utilizó la técnica de encuesta mediante un cuestionario. Este instrumento fue aplicado a 245 personas a través de un muestreo de casos-tipo, seleccionadas por su rango de edad de entre los 20 y 35 años y por haber realizado viajes vacacionales en el año anterior a la investigación. La zona de estudio fue la ciudad de Villahermosa, capital del estado de Tabasco, México. Los resultados muestran que este segmento prefiere visitar sitios turísticos culturales, que la elección del destino forma parte de una decisión familiar y que sus salarios no son muy elevados, lo anterior deberá ser tomado en consideración como un referente para los prestadores de servicios turísticos y demás actores sociales involucrados en el desarrollo de este segmento.}

Palabras clave: millennials, mercadotecnia, consumo turístico.

\begin{abstract}
:
The objective of the article is to identify the factors that influence the choice of a tourist destination in the generation known as millennials. The methodological approach presented is quantitative, it used the survey technique through a questionnaire. This instrument was applied to 245 people through a sample of typical cases, selected for their age range of between 20 and 35 years and for having made vacation trips in the year prior to the investigation. The study area was the city of Villahermosa, capital of the state of Tabasco, Mexico. The results show that this segment prefers to visit cultural tourist sites, that the choice of destination is part of a family decision and that their salaries are not very high, this should be taken into consideration by tourism service providers and other social actors involved in the development of this segment.
\end{abstract}

Keywords: millennials, marketing, tourism consumption. 


\section{INTRODUCCIÓN}

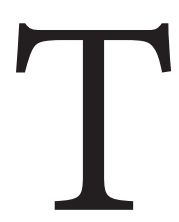
abasco es uno de los estados que conforman la República Mexicana, su capital es la ciudad de Villahermosa. Esta ciudad se ha destacado por ser un centro de negocios y administración de la industria petrolera, sin embargo este sector económico ha sufrido importantes cambios en la última década, lo que ha propiciado una importante disminución de empleos en toda la entidad. Lo anterior lo ha dado a conocer el Instituto Nacional de Estadística y Geografía (INEGI, 2020) a través de sus reportes trimestrales relacionados con la desocupación total en los diferentes estados del país. En el primer trimestre del año 2020 se ha registrado en Tabasco un 7,4\% de población desocupada. Ante dicha situación, la actividad turística representa una alternativa para la dinamización de la economía local.

El estado de Tabasco se caracteriza por poseer una diversidad de atractivos turísticos del orden natural y cultural. La oferta turística más representativa incluye productos relacionados con el cultivo del cacao y la producción del chocolate artesanal, aviturismo y pesca deportiva, así como los festivales regionales; particularmente aquellos relacionados con la promoción de la gastronomía típica. Entre ellos destaca el festival del chocolate que se ha realizado desde el año 2010 con la finalidad de "elevar la estacionalidad de la demanda en el mes de noviembre, para crear cadenas de valor en torno al cacao e internacionalizar sus productos derivados" (Vidal, 2017, p.501).

Se considera importante dirigir los esfuerzos a aquellos segmentos de mercado que realmente puedan impulsar la economía tabasqueña. Esto hace obligatorio el análisis de los millennials debido a que pronto llegarán a ser la principal fuerza de trabajo. Para Madrigal, Madrigal y Ávila (2019, p.87), "los millennials representan un importante capital creativo, dinámico y tecnológico crucialmente necesario en la organización de vanguardia”. Por otro lado, Posso y Cardona (2016, p.149) describen a los millennials como una generación totalmente diferente $\mathrm{y}$ revolucionaria a cualquier otra generación ya existente, esto por ser personas nacidas en época de cambios profundos y sobre todo en una era digitalizada donde la tecnología está al alcance de todos y facilita ciertas actividades de la vida cotidiana.

La generación millennials ha sido fuente de algunos estudios y debates a nivel mundial, ya que se han estado convirtiendo en un segmento de potenciales consumidores. Por lo que muchas de las investigaciones realizadas recalcan la importancia de comprender a estos jóvenes consumidores haciendo lo posible por crear productos que realmente puedan consumir.

Para la creación de productos turísticos es necesario tener presentes los resultados del estudio de Posso y Cardona (2016, p.153) que señalan que los millennials que más viajan son del género masculino y una característica de esta generación es que al momento de viajar lo hacen solos, por cuenta propia y sin necesidad de una agencia para organizar sus viajes. Son personas que se encuentran cursando la universidad o cuentan con un trabajo que les permite obtener buenos ingresos si se encuentran solteros e invertir en una estadía de 1 a 6 días en el destino que visitan. 
En todo el mundo se puede observar el crecimiento en el consumo de viajes efectuados por esta generación, aun cuando muchos de ellos se encuentran iniciando su vida adulta. Esto impulsa la búsqueda de más información sobre este grupo de personas, información que ayude a comprender más sobre sus gustos y preferencias. Por lo tanto, el objetivo del presente estudio es identificar los factores que influyen en los millennials para la elección de un destino turístico. El conocimiento de estos factores y sus repercusiones abonarán al sector turístico tabasqueño, ya que esta información podrá tomarse como un marco referencial para el diseño de productos que sean capaces de satisfacer los gustos y preferencias de este importante segmento de mercado para atraer visitantes nacionales y extranjeros.

\section{REVISIÓN DE LITERATURA}

Actualmente la industria turística está atravesando por diferentes cambios, esto es perceptible en el "desarrollo de nuevos tipos de turismo, transformaciones en la demanda, influencias en la tecnología, surgimiento de nuevos destinos y mercados emisores" (Silveira y Silveira, 2009, p.530). Además, en el último par de años, la pandemia de la COVID-19 llegó a exacerbar la necesidad de elevar la calidad de la oferta turística; especialmente en los controles sanitarios, tanto en la infraestructura como en la prestación del servicio. Estos cambios acelerados obligaron a todo el sector turístico a mantenerse a la expectativa por cualquier acontecimiento o tendencia que surja de manera inesperada para tomar ventaja y ofrecer alternativas viables a la oferta y demanda turística.
Por otro lado, el fenómeno turístico requiere de un espacio geográfico que cuente con ciertas características físicas, sociales o ambientales atractivas para los seres humanos. Estos espacios o destinos turísticos "no son otra cosa que territorios donde se encuentran determinados componentes de una oferta turística estructurada a partir de atractivos, productos, servicios, accesos y demás elementos que hacen posible una estancia" (Rodríguez, 2011:1).

Todo destino turístico cuenta con sus elementos involucrados como lo son los elementos tangibles y los elementos intangibles. Los elementos tangibles se encuentran integrados por todos aquellos elementos que se pueden ver y tocar, tales como los atractivos turísticos naturales y culturales, infraestructura y equipamiento, servicios turísticos y recursos humanos, mientras que los componentes intangibles no se pueden ver $y$ tocar pero si se perciben y se transforman en experiencias. Dentro de estos elementos se pueden encontrar la seguridad, la imagen, identidad local y la información e interpretación (SERNATUR, 2016, p.15).

Tradicionalmente, "en el turismo se destacan cuatro actores que poseen una gran capacidad para influir y configurar el territorio: la comunidad local, el sector público, el sector privado y los turistas" (Moscoso, 2013:8). La comunidad local es la que se encuentra integrada por los habitantes de la localidad considerada destino turístico y que de alguna manera se ve beneficiada o perjudicada por el desarrollo de esta actividad dentro de sus límites geográficos. El sector público debido a que se encarga de generar la agenda de las políticas públicas encaminadas a impulsar el desarrollo turístico de la región o del país. El sector privado 
integrado por empresas que se benefician del turismo de manera directa o indirecta; y por último, los turistas que se trasladan al lugar de su preferencia para disfrutar de los elementos del destino turístico.

De acuerdo a Chirinos (2009, p.140) los millennials son individuos nacidos entre los años 1982-2000 y se caracterizan por ser idealistas, estar conectados las 24 horas los 7 días de la semana, tener un pensamiento social y activo, respeto por el prójimo, orientado al logro, la búsqueda de la mejor oferta de dinero, más creativo, entre otras. Es necesario mencionar que gran parte de los consumidores turísticos corresponden a la generación que ha sido denominada millennials, quiénes para el año 2025 sostendrán el consumo en todas las áreas ya que representarán el 75\% de la fuerza laboral (Ceballos, 2017).

En un estudio realizado por Peñalosa y López (2016) denominado "la generación de los millennials frente al consumo socialmente responsable" se muestra con claridad sus preferencias relacionadas al mismo. Este estudio fue aplicado a jóvenes de clase media entre 18 y 25 años que habitaban la ciudad de Bogotá, Colombia. La actitud positiva frente al consumo socialmente responsable es uno de los principales hallazgos del estudio ya que la tendencia de la población estudiada muestra que se preocupan por el medio ambiente, el uso y desecho de los productos, además de poseer dentro de sus rasgos de personalidad una conciencia medioambiental bien definida.

Por lo anterior, dentro de poco, será necesario la evaluación de los destinos turísticos para determinar su sostenibilidad, relacionada al equilibrio obtenido en los tres ejes que la forman: social, económico y ambiental. En el eje social se deberá medir el nivel de satisfacción e integración de los habitantes del destino turístico a las diferentes acciones que se realizan para impulsar el turismo de la localidad así como los beneficios sociales derivados de estas actividades. Mientras que en el eje económico se debe de poner especial énfasis al nivel de ingreso percibido dentro de la localidad así como la calidad de vida desplegada por sus integrantes. Por otro lado, en el eje ambiental se pondrá especial énfasis en las políticas, estrategias y acciones destinadas a conservar todo el patrimonio natural existente en el lugar y sus alrededores (Saeteros, Da Silva y Flores, 2019). Lo anterior será de vital importancia para poder alcanzar la preferencia de los actuales consumidores turísticos que basan sus preferencias sobre los valores humanos, comportándose con responsabilidad y sumamente sensibles a las tendencias que se encargan de cuidar o mejorar su entorno por lo que no dudarían en cambiar sus preferencias por destinos turísticos que cumplan con esta demanda.

Los millennials son un segmento de la población que, además de ser más sensibles al cuidado medioambiental, también participan activamente en las redes sociales dominando diferentes aplicaciones tecnológicas que facilitan sus actividades diarias por lo que la gran parte de su día se encuentran conectados a internet a través de sus diferentes dispositivos y computadoras. Aman viajar y tener excelentes experiencias en sus viajes. Es así que "el 74\% de los millennials, por ejemplo, busca información de viajes en sus dispositivos móviles y el $49 \%$ realiza sus reservaciones a través de este canal, representando 
casi el doble que el resto de los compradores online; mientras que el $90 \%$ de los millennials consulta internet para planear sus viajes en una PC” (Ceballos, 2017, p.1).

De acuerdo con un estudio realizado por Roselló (S/F) esta generación viaja una vez cada tres o seis meses y prefiere hacerlo en la época de verano. Para esta generación es importante planificar con tiempo sus viajes, ya que de esta manera pueden conseguir mejores precios, ubicaciones, disponibilidad y programar las actividades al destino seleccionado. Los millennials prefieren hacer sus viajes dentro de su propio país o dentro del continente, ya que buscan destinos en donde puedan encontrar espacios culturales, monumentos, museos y templos aunque también optan por destinos de playa $y$ lugares en donde puedan involucrarse en actividades de aventura conviviendo a la vez con la naturaleza. Además buscan economizar en alojamiento y les encanta compartir experiencias con otros viajeros a través de las redes sociales, han marcado la diferencia con las generaciones pasadas ya que ellos han crecido y evolucionado con la tecnología. Para esta generación las redes sociales son indispensables además que de ahí buscan a sus proveedores encontrando así servicios fidedignos con opiniones y experiencias de otros usuarios que ya han realizado viajes al destino que desean viajar.

Desde otra perspectiva, Blázquez, Collado y Talaya (2004) revelan que estos jóvenes utilizan distintas fuentes de información. Estas pueden ser personales e impersonales como familiares, amigos, guías, folletos y hasta de comentarios de desconocidos en las diferentes redes sociales; representando para ellos mayor importancia, credibilidad e influencia directa al momento de elegir un destino turístico. Además, estos autores sustentan que la utilización de las fuentes de información no depende de las características del consumidor en su condición de turista, sin embargo, el número de fuentes consultadas si depende de las características de los turistas.

Sin embargo para Posso y Cardona (2016), los medios de comunicación juegan un papel importante a la hora de obtener información acerca del destino al que planean viajar, ya que la mayoría prefiere hacer sus búsquedas por medio de internet y redes sociales. Por este motivo los destinos y sitios de interés turísticos deben estar preparados ante las preferencias de estos nuevos consumidores, por lo que deben contar con una página web vanguardista donde pueda existir una interacción y un plan de marketing en línea que les permita estar a la vanguardia con esta generación. Para aprovechar el potencial de consumo turístico de estas generaciones es importante realizar más estudios y diagnósticos sobre esta población y a su vez motivar a los destinos o empresas de servicios turísticos para que generen campañas de promoción que muestren las características únicas del atractivo para que impacten positivamente a los millennials. Además, de acuerdo a estos autores, es necesario diseñar estrategias que permitan aumentar y mejorar la cultura hospitalaria de los ciudadanos de un destino turístico para hacer más grata la visita de los turistas creando experiencias inolvidables y generando el deseo de volver.

Por otro lado, Quevedo y Pérez (2018) realizaron un estudio en México para identificar las preferencias turísticas y gastronómicas de los millennials. El instrumento de recolección de datos 
fue aplicado a través de las redes sociales debido a que la población estudiada, como se sabe, ellos son nativos digitales que siempre están conectados a las plataformas tecnológicas. Los resultados relacionados a las preferencias turísticas reflejan el marcado uso de medios electrónicos, computadora y celular, para la realización de las reservaciones de viaje, además la planeación para el mismo les toma de uno a seis meses prefiriendo ante todo, los destinos nacionales a los internacionales. En cuanto al consumo gastronómico, los resultados reflejan su marcada predilección por la comida personalizada. Consumen al menos tres veces por semana los alimentos fuera de casa, registrando que esto lo hacen por placer aunque en ocasiones las condiciones de trabajo les orillan a visitar establecimientos de comidas.

Como se ha podido observar, aun cuando los millennials realizan todas sus tareas y consultas a través de medios digitales no dejan de comportarse como lo hacen los consumidores tradicionales. El comportamiento del consumidor, Schiffman y Kanuk (2010, p.5) lo definen como aquel "que los consumidores exhiben al buscar, comprar, utilizar, evaluar y desechar productos y servicios que ellos esperan que satisfagan sus necesidades".

Actualmente todo consumidor requiere lidiar con las estrategias de mercadotecnia dirigidas a ellos para motivarlos a realizar las compras. Es aquí donde entra el proceso de decisión por lo que para examinarlo es importante analizar las fuerzas primarias que influyen el comportamiento de compra (Kotler y Armstrong, 2008). En la adquisición de los diferentes productos y servicios ofertados en el mercado los factores que más influyen son aquellos relacionados a los aspectos culturales, personales, sociales y psicológicos (Ponce, Besanilla y Rodríguez, 2012).

Los factores culturales son los que ejercen una gran influencia en el consumo de los individuos derivado de que la cultura "es un conjunto de valores, percepciones y comportamientos que un miembro de la sociedad aprende de su familia o de otras instituciones importantes" (Kotler, 2010, p.10). Este modo de vida incluye los valores de cada país, región o localidad que se manifiestan en el día a día a través de diferentes patrones de consumo, educativos, residenciales, entre otros. Es bajo este contexto cultural que se formulan diferentes productos y servicios para integrar la oferta actual en el mercado. El factor personal es otro aspecto que debe de tenerse presente ya que los hábitos de compra y las decisiones de cada individuo se encuentran claramente influidos por la edad y la etapa en el ciclo de vida en la que se esté transitando, el estado civil que se ostente, la ocupación, el nivel de gastos y ahorros, las actividades que realiza, sus intereses, su personalidad, su percepción, entre otras (Ponce, 2012).

Los factores sociales influyen en el comportamiento de compra de los consumidores porque se proyectan imágenes en los grupos en los que se integran definiendo el estatus al que pertenecen. Por lo que "la originalidad que pueda tener cada ser humano diferenciándose de los demás individuos, siendo siempre el primero en probar tal producto o marca de la empresa, o también usar productos ostentosos que despierten la envidia del resto del grupo" (Ruiz y Rodríguez, 2018:25) influirá en la elección de destinos turísticos. 
Por último, se tienen los factores psicológicos, es decir aquellos estrechamente ligados a las creencias y actitudes que influyen de manera directa en nuestra decisión de compra para obtener reconocimiento, estima o pertenencia por parte de sus semejantes. El factor psicológico “determina que el modelo de compra está ligado directamente con la individualidad de cada sujeto, pudiendo ser determinado por la motivación, percepción, aprendizaje, creencias y actitudes" (Possebon, Cervi y Knebel, 2019, p.906). Sin embargo el ser humano necesita salud mental y sentirse realizado. Estas necesidades surgen del factor psicológico vinculado con los aspectos que las personas requieren para sentirse amados, reconocidos y estimados, tanto en lo personal como en lo profesional.

\section{METODOLOGÍA}

El estudio parte de un enfoque metodológico cuantitativo que intenta describir las razones que tienen los viajeros millennials para escoger destinos turísticos. Los sujetos observados fueron personas comprendidas en un rango de edad de entre los 20 y los 35 años, que al compartir características culturales similares pertenecen a una generación llamada millennials. Siendo uno de los factores de elección el que hayan realizado viajes vacacionales en el año anterior a la investigación. La muestra se determinó con un porcentaje de confiabilidad del 95\% y un margen de error del 6\%, dando como resultado una muestra de 245 personas. Los cuestionarios se aplicaron a 118 hombres y 127 mujeres en diferentes parques de la ciudad de Villahermosa, como el Parque Tomás Garrido Canabal, la Pólvora y Parque la Choca durante 4 fines de semana consecutivos. Del total de la población estudiada el $77 \%$ cuenta con un empleo estable, mientras que el $23 \%$ cuenta con trabajos esporádicos.

Las categorías analizadas durante la investigación se fundamentan en cuatro aspectos del comportamiento humano: culturales, sociales, económicas y psicológicas. Se diseñó un cuestionario integrado por 16 preguntas con opción de respuesta múltiple (Véase Tabla 1, próxima página).

En el factor cultural, es decir, los modos de vida que se expresan al momento de desempeñar diferentes papeles dentro de la comunidad, se realizaron cuatro preguntas, a fin de evaluar las motivaciones culturales del viaje, los tipos de destinos preferidos, la pertenencia a alguna tribu urbana y el reconocimiento de la tribu urbana a la que pertenece. Respecto a las motivaciones culturales para viajar se presentaron como opciones de contestaciones: el incremento de conocimientos, conocimiento de otros modos de vidas, exploración de otros lugares y vivir nuevas experiencias. Por otra parte, la preferencia de destinos dio como posibles respuestas: playas, pueblos mágicos, lugares históricos y culturales y destinos de naturaleza.

La tercera y cuarta pregunta permitieron identificar si dentro de este segmento de población se encontraban individuos que pertenecieran a alguna tribu urbana, es decir "pandillas, bandas o simplemente agrupaciones de jóvenes y adolescentes que se visten de modo parecido y llamativo, siguen hábitos comunes y se hacen visibles" (Pere-Oriol, Pérez y Tropea, 1996, p. 11). Entre las opciones presentadas estuvieron los hípster, éstos para Fauce y Pedro (2016, p.154) 
Tabla 1.

Categorías e indicadores analizados en los millennials.

\begin{tabular}{ll}
\hline CATEGORÍAS & INDICADORES \\
\hline I. Cultural & 1. Motivaciones culturales del viaje \\
& $\begin{array}{l}\text { 2. Tipos de destinos preferidos } \\
\text { 3. Pertenencia a alguna tribu urbana } \\
\text { 4. Reconocimiento de la tribu urbana a la que } \\
\text { pertenece }\end{array}$ \\
5. Reconocimiento de la clase social a la que \\
pertenece \\
6. Influencia directa de personas en la elección del \\
destino turístico \\
7. Fuentes de información consultadas para la \\
planificación del viaje
\end{tabular}

"son acusados de elitistas: su consumo cultural no es sino una manera de marcar diferencias entre ellos y otros grupos sociales que no poseen el mismo capital cultural". Luego la comunidad LGBT que para Castellanos (2016, p.10) su libertad ha sido restringida limitando el ejercicio pleno de su ciudadanía. También se incluyó como opción a los muppies, que para Sánchez (2017) son personas que "trabajan en lo que les gusta, son adictos a las redes sociales pero su verdadero motor son el deporte y la ingesta de alimentos saludables". Así como a los dark, que son personas cultas, refinadas, conocen de cine, música y literatura y acostumbran a vestir de color negro (Escribano y Carrera, 2008).
Para lo que podría denominarse como factores sociales que influyen en sus decisiones, se realizaron tres preguntas. La primera hace referencia a su percepción sobre la clase social a la que pertenece. De acuerdo a Gutiérrez (2004), la clase social baja se caracteriza por individuos con un nivel educativo básico y viviendo en hogares de interés social o rentas congeladas; la clase social media se caracteriza por tener sus propias casas o departamentos con algunas comodidades; mientras que la clase social media alta vive en casas o departamentos lujosos, y por último; la clase social alta se caracteriza por contar con niveles educativos superiores viviendo en casas o departamento lujosos, con todas las comodidades. 
Dentro de los factores sociales evaluados, se encuentra la clase social.

La segunda pregunta de esta categoría abordó la influencia directa que tienen ciertas personas, como compañeros, familiares, amigos y pareja, sobre la selección del destino turístico. Además la categoría incluyó un cuestionamiento sobre las fuentes de información consultadas al planificar un viaje.

Para identificar la situación económica de los millennials se realizaron seis preguntas. La primera pregunta buscó identificar el tipo de alojamiento preferido por este segmento de mercado. También se incluyeron preguntas sobre las condiciones laborales, como la dependencia económica, la realización de una actividad remunerada y los ingresos percibidos. Los últimos dos indicadores de esta categoría evaluaron el consumo turístico y la estancia promedio realizada en los destinos turísticos.
En lo que se puede denominar factor psicológico, que involucra el aprendizaje, las creencias y actitudes, se aplicaron tres preguntas. La primera acerca de las prioridades a considerar al momento de elegir un destino turístico: ubicación, precio, seguridad y posicionamiento o reconocimientos del lugar. La segunda cuestiona sobre las expectativas que se pueden tener del viaje: diversión, relajación, nuevas relaciones y aumentar estatus social. Finalmente se preguntó respecto a la relación existente entre la personalidad de los entrevistados con el tipo de comportamiento turístico.

\section{ANÁLISIS DE LOS RESULTADOS}

Respecto al factor cultural de los encuestados, se encontró que la mayoría decide viajar para aprender sobre la cultura de otros lugares, seguido por el motivo de vivir nuevas experiencias, incrementar conocimientos $y$, por último, para conocer otros modos de vida (Véase Figura 1).

\section{Figura 1.}

Motivaciones culturales de viaje en los millennials.

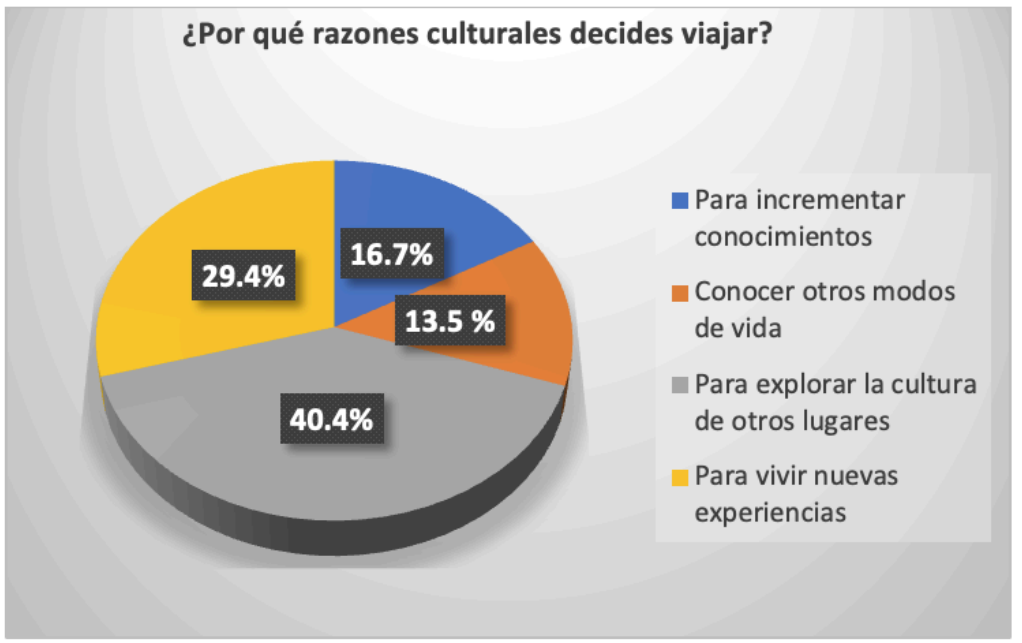

Fuente: Elaboración propia. 
Los tres sitios o destinos turísticos que cuentan con mayor preferencia en el mercado de los millennials son en su mayoría los lugares con importancia cultural, destinos de naturaleza $y$ pueblos mágicos. Se infiere con estos datos que las motivaciones de viaje de este segmento están dirigidas a la práctica de actividades turísticas vinculadas con las tradiciones, folklore, vestimenta típica entre otras, así como destinos donde prevalecen los espacios abiertos, en entornos naturales o rurales, alejados de la ciudad y de la cotidianeidad. Finalmente un $19.6 \%$ prefiere viajar a los destinos tradicionales de playa (Véase Figura 2).

Figura 2.

Destinos preferidos por los millennials.

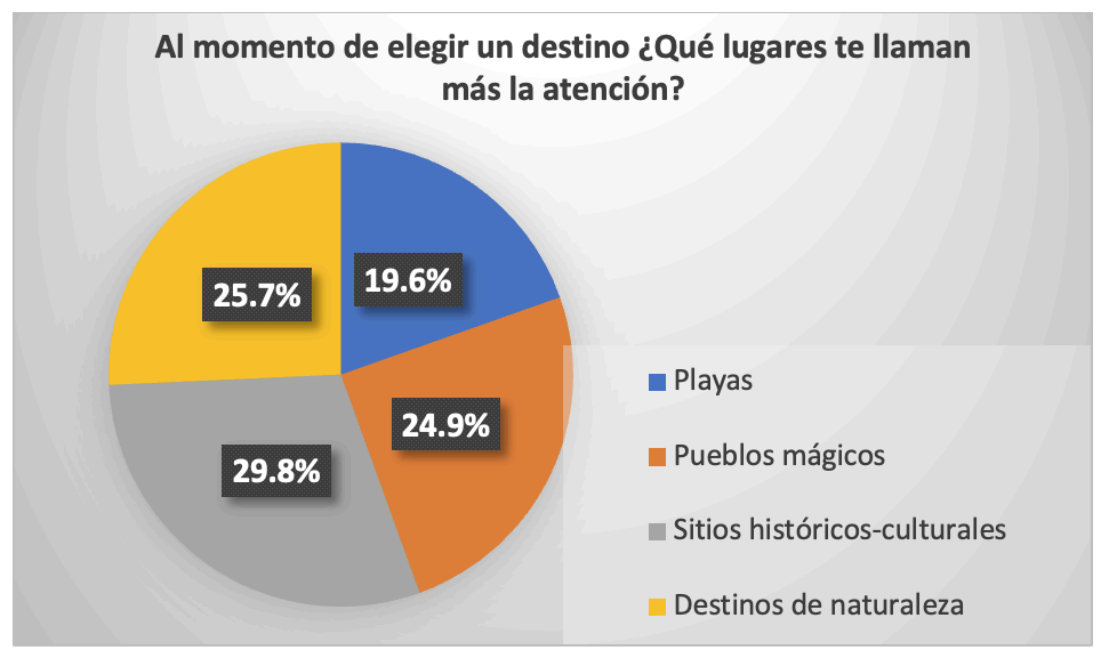

Fuente: Elaboración propia.

Los millennials constituyen una generación que integra a diferentes tribus urbanas. En el caso de los encuestados, el $20.4 \%$ se identificó con alguna de éstas tribus. De este porcentaje, la mayoría se asume como hípster, seguidos por la comunidad LGBT, los dark y los muppies (Véase Figura 3). 


\section{Figura 3.}

Tribus urbanas de los millennials.

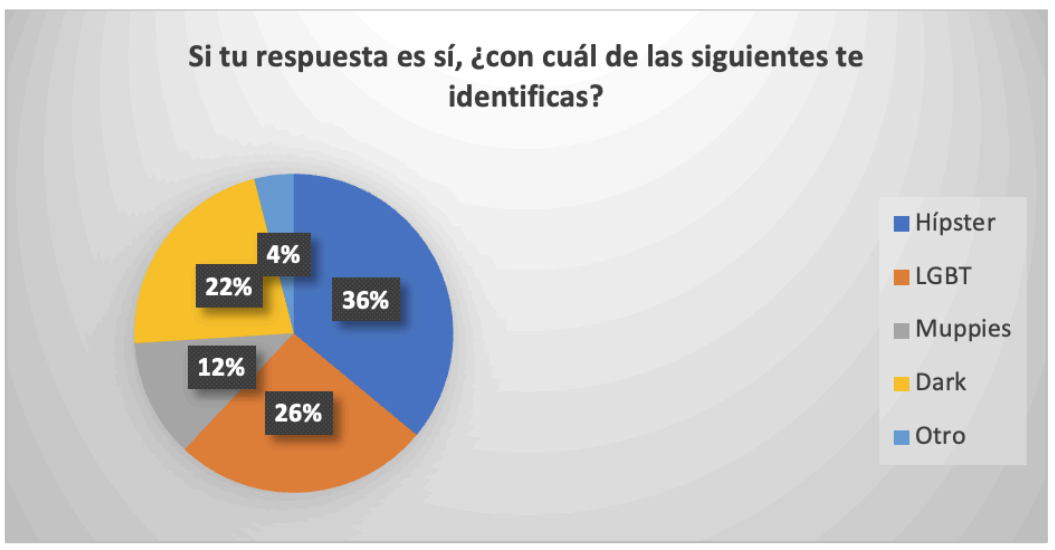

Fuente: Elaboración propia.

Al medir la clase social en la que cada individuo se percibe, se obtuvo como resultado que la gran mayoría dijo pertenecer a la clase media, con $76.7 \%$. Sólo una persona consideró pertenecer a la clase alta (Véase Figura 4).

Figura 4.

Clases sociales de los millennials.



Fuente: Elaboración propia.

Conforme a la influencia directa de personas en la elección de los destinos turísticos, se observó que poco más de la mitad de los encuestados es influenciado por la familia, en un $27.3 \%$ por los amigos y en un $14.7 \%$ por la pareja (Véase Figura 5). 


\section{Figura 5.}

Influencia directa de personas en los millennials durante la elección del destino turístico.

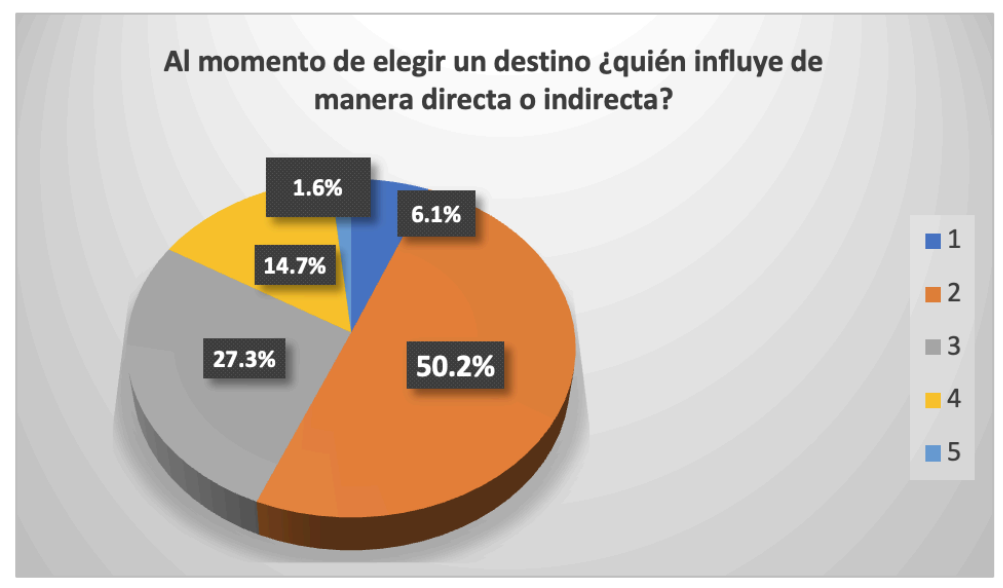

Fuente: Elaboración propia.

Por otra parte, la fuentes de información más utilizadas por los millennials, al momento de planificar un viaje indican que la mayoría consulta con familiares y amigos, seguido por las redes sociales para obtener información, páginas de viajes y en menor medida utilizan a las agencias de viajes tradicionales como intermediarios (Véase Figura 6).

\section{Figura 6.}

Fuentes de información consultadas para la planificación del viaje por los millennials.

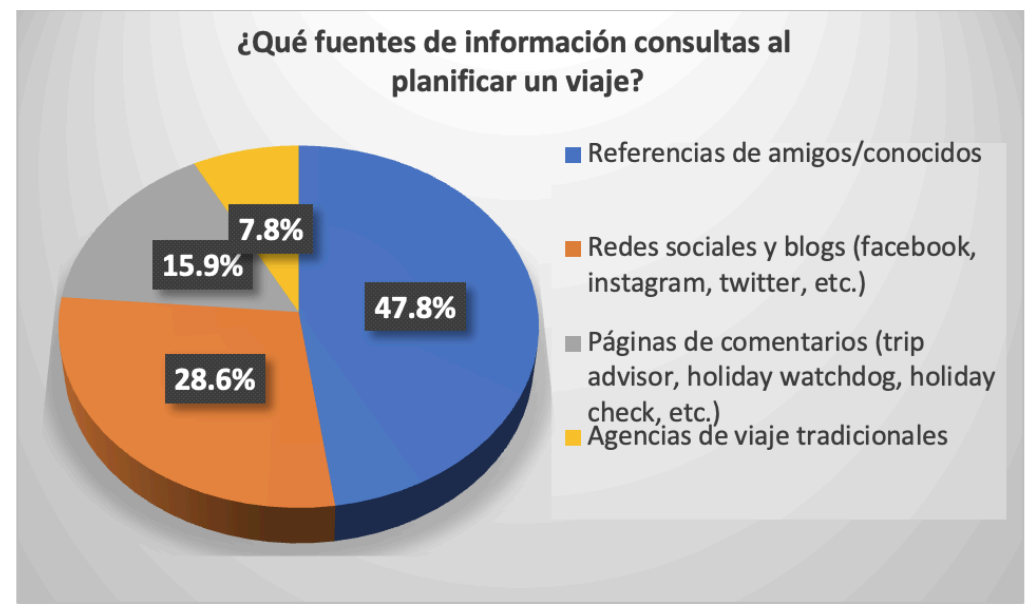

Fuente: Elaboración propia.

Respecto a la situación económica de los millennials y su relación con el gasto turístico, se observó que la gran mayoría, 75.9\%, prefiere hospedarse en un hotel económico. En contraste, solo el $8.2 \%$ prefiere un hotel de lujo y el resto de los encuestados seleccionaron establecimientos de hospedaje del tipo extra-hotelero, es decir, establecimientos con facilidades de alojamiento limitadas, como hostales y casa de huéspedes (Véase Figura 7). 


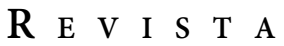 \\ Public a n d o \\ I S S N $13390-9304$}

\section{Figura 7.}

Preferencia de hospedaje de los millennials.

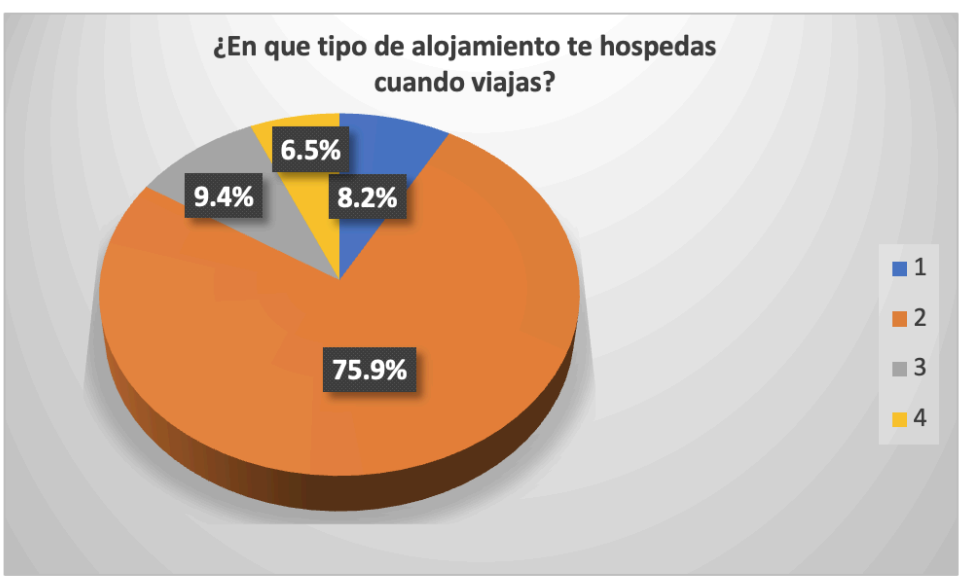

Fuente: Elaboración propia.

Además se observó que la mayoría de los millennials, 61.6\%, dependen de ingresos propios, mientras que un $28.2 \%$ dependen económicamente de su familia (Véase Figura 8).

\section{Figura 8.}

Dependencia económica de los millennials.



Fuente: elaboración propia.

El 77\% de los participantes aseguró tener un empleo remunerado. De estos, un $46.1 \%$ señaló tener ingresos de $\$ 200$ a $\$ 400$, un $33.9 \%$ dijo tener ingresos de menos de $\$ 200$, un $16.3 \%$ con ingresos de $\$ 400$ a $\$ 750$ y un $3.7 \%$ con ingresos de más de $\$ 750$. Por otra parte, el consumo turístico informado indica que un $47.8 \%$ gastan de entre \$100 y \$250 (Véase Figura 9). 
Figura 9.

Consumo turistico de los millennials.

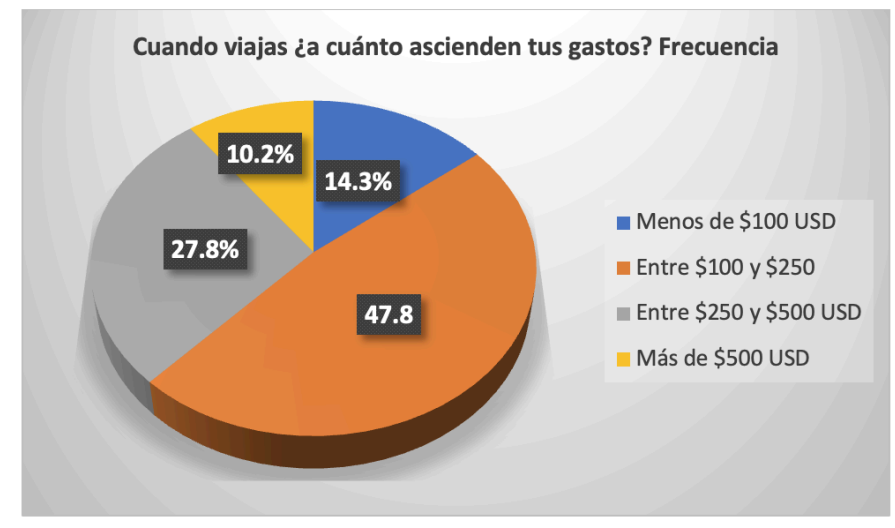

Fuente: Elaboración propia.

El $50.2 \%$ de los millennials encuestados mencionaron que la estancia promedio que realizan en los destinos turísticos oscila de 3 a 5 días, un $26.5 \%$ de menos de 3 días, el $18.8 \%$ de 5 a 7 días y el $4.5 \%$ tienen una estancia de más de una semana.

En cuanto al análisis del factor psicológico como determinante al momento de elegir un destino de viaje entre este grupo demográfico, la encuesta arrojó que los aspectos más relevantes son el precio, seguido por la ubicación. Cabe resaltar que la seguridad del destino representa tan sólo el $19.6 \%$ de los aspectos a considerar en la selección del destino turístico (Véase Figura 10).

Al cuestionar sobre las expectativas o motivos que se tienen sobre el destino turístico, el 47.3\%, de los encuestados eligió la opción de divertirse al máximo y un $44.1 \%$ la relajación. En menor medida se observó que los millennials hacen viajes con la intención de hacer nuevas amistades y aumentar su estatus social, $\quad 5.7 \%$ y $\quad 2.9 \%$ respectivamente.

\section{Figura 10.}

Prioridades de los millennials en la selección del destino turístico.

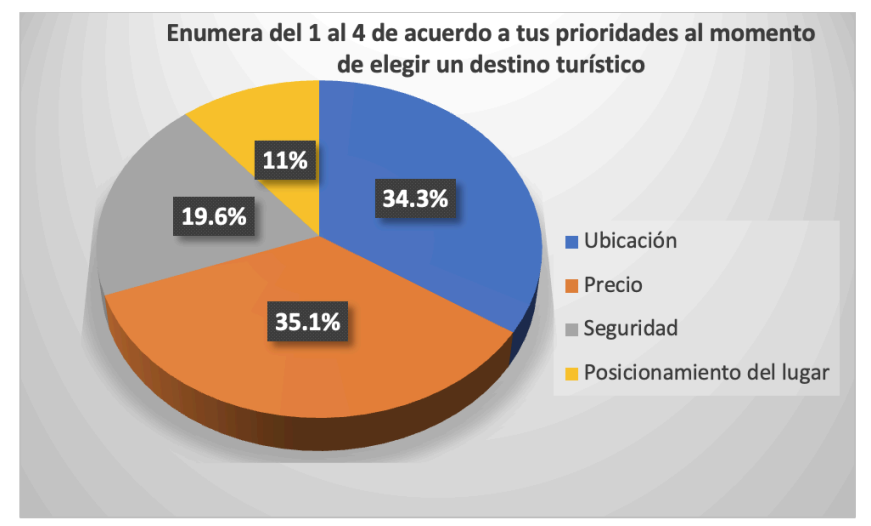

Fuente: Elaboración propia. 
Finalmente, respecto a la autopercepción de los rasgos de personalidad con relación al tipo de consumo turístico, el $50.6 \%$ de los millennials se percibe como personas que prefieren pagar directamente en el destino, usan muy poco las agencias de viajes en la planeación de sus vacaciones, tienen poco interés por el confort y usan información factible y descriptiva. El 39.2\% se perciben como personas que prefieren pagar los servicios de una agencia de viajes, les importa mucho la comodidad y les gusta usar información focalizada. El 10.2\% dicen ser personas que tienen poco interés en toda materia ecológica y en el transporte limpio, son personas pasivas que tienen expectativas reducidas y son personas prácticas.

\section{DISCUSIÓN}

Esta investigación ofrece información relevante sobre los estilos de consumo turístico de los millennials en Tabasco, México. Uno de los resultados más interesantes fue detectar la preferencia de este segmento por los aspectos culturales al elegir un destino turístico. Este resultado contrasta con la idea que se tenía sobre la preferencia de destinos de playa nacionales e internacionales (Cornelio y Cuevas, 2019) ya que esta generación no siempre busca diversión sino una nueva forma de aprendizaje.

Por otro lado, los resultados relacionados con el factor social, al igual que el estudio desarrollado por Blázquez (2004), muestran que los millennials al momento de elegir un destino turístico, aun cuando las redes sociales y la tecnología son sus herramientas diarias, la familia y las referencias sobre los lugares turísticos visitadas por amigos juegan un papel muy importante en sus elecciones, debido a que otorgan mayor credibilidad a la información que les proporcionan de manera directa.

El factor económico muestra a un grupo de poco poder adquisitivo y con pocas posibilidades de pagar servicios turísticos de costos elevados, este resultado muestra un severo contraste con los hallazgos del estudio realizado por Posso y Cardona (2016) donde midieron las tendencias de consumo turístico de los millennnials en la ciudad de Ibarra, encontrando millennials solteros, que cuentan con un trabajo que les permite obtener buenos ingresos.

Por último, el factor psicológico muestra que la mayoría de los encuestados se consideran personas que prefieren pagar directamente en el destino, usan muy poco los servicios de las agencias de viajes para planificar sus vacaciones y tienen poco interés por el confort. Solo buscan comprar, utilizar, evaluar y desechar productos que satisfagan sus necesidades, tal y como Shiffman (2010) ha calificado el comportamiento del consumidor.

Por lo que la situación particular, observada en Tabasco, debe ser un parteaguas que tome en cuenta las condiciones actuales de los millennials en el estado, que dirija a los prestadores de servicios turísticos en la organización de opciones de productos de viaje de bajo costo que propicie el consumo de insumos locales, que eleve la demanda, y con ésta, la retribución económica para el sector turístico sea satisfactoria.

\section{CONCLUSIONES}

Los millennials son personas conectadas con un mundo tecnológico que es de gran apoyo al momento de dar y obtener información para tomar decisiones. Sin embargo es necesario dejar 
claro que existen factores que son determinantes al momento de elegir una opción. Estos factores se encuentran relacionados directamente con el ámbito cultural, social, económico y psicológico que rodean a cada uno de los integrantes de este segmento por lo que si se desea atraer más turismo a territorio tabasqueño es indispensable tomar en cuenta la información generada a través de este estudio.

Por los resultados expuestos se recomienda a los prestadores de servicios turísticos del Estado que hagan un análisis profundo y detallado de sus costos, servicios ofrecidos, actividades turísticas, canales de distribución y promoción para la atracción de la oferta turística. Este análisis podrá permitir la combinación correcta de los gustos y preferencias de la población estudiada y así llegar a este segmento de manera contundente a través de estrategias correctamente dirigidas para propiciar su desplazamiento turístico a destinos que han considerado más relevantes como los culturales, $29.8 \%$, en donde pueden encontrar tradiciones, folklore, vestimenta típica entre otras; los de naturaleza, $25.7 \%$, pueblos mágicos, $24.9 \%$ y por último, los de playa, $19.6 \%$.

La implementación de productos turísticos innovadores, diseñados a través de la información obtenida del análisis de este segmento de mercado turístico, asegurará la rentabilidad y los beneficios económicos para los empresarios turísticos y la población local, además de influir en la percepción de los turistas, al encontrar experiencias que enriquecerán sus conocimientos sociales y ambientales, y totalmente de acuerdo a su capacidad de gasto.

Por otro lado, los resultados aquí obtenidos contribuirán a que el sector turístico de Tabasco aproveche y fortalezca otras actividades en las regiones en donde se implementen, "procurando la creación de cadenas productivas para apoyar la consolidación de una economía balanceada" (COPLADET, 2019). 


\section{REFERENCIAS}

Blázquez, J.J., Molina, A., Esteban, A. y MartinConsuegra, D. (2008). Análisis de la eficacia publicitaria en Internet. Investigaciones Europeas de Dirección y Economía de la Empresa, vol. 14, num.1, 159-176. https:// www.researchgate.net/publication/ 28216597_Analisis_de_la_eficacia_publicita ria_en_internet

Castellanos, C. (2016). La apropiación del espacio público, de las personas de los sectores de lesbianas, gays, bisexuales y transgeneristas (LGBT) en la localidad de Chapinero, a partir de la expedición de la política pública LGBT. [Tesis Maestría, Universidad Piloto de Colombia]. http://repository.unipiloto.edu.co/ b i t s t r e a m / ha n d l e / 20.500.12277/3312/00003280.pdf?sequence= 1\&isAllowed=y

Ceballos, Francisco (27 de febrero de 2017). Los millennials redefinen el rumbo del turismo. Red Forbes México. https:// www. forbes.com.mx/millennials redefinen-el-rumbo-del-turismo/.

Chirinos, N. (2009). Características generacionales y los valores. Su impacto en lo laboral. Observatorio laboral revista venezolana, 2(4), 133-153. https://www.redalyc.org/pdf/ 2190/219016846007.pdf

COPLADET (2019). Programa Sectorial de Desarrollo turístico 2019-2024. Comisión de Planeación para el Estado de Tabasco. h t t p : / / ve.s c i e lo.org / scielo.php?script=sci_arttext\&pid=S0798 $-07522004000100004$
Cornelio, B.L. y Cuevas, M.C. (2019). Marketing Turístico para Millenniass. Revista Hitos de Ciencias Económico Administrativas, Año 25, Número 71, pp 36-49. https:// revistas.ujat.mx/index.php/hitos/article/ view/3601/2736

Escribano, M. y Carrera, M. (2008). Soy diferente. Emos, Darketos y otras tribus urbanas: Una guía para entender a los adolescentes de hoy. México, ed. Diana.

Fouce, H. y Pedro, J. (2017). Hípsters, ¿alternativos o masivos? Límites de la controversia en la esfera pública cultural. En ÁlvarezPeralta, Fernández L.M. (Eds), la mediación fragmentaria Mediatización y controversia en la nueva esfera pública (132). Cuadernos Artesanos de Comunicación- Creative Commons. http://cuadernosartesanos.org/2017/ cac132.pdf $\#$ page $=147$

Gutiérrez, F. (2004). Clasificación de niveles socioeconómicos en México según la AMAI. Fergut. https://www.fergut.com/clasificacionde-niveles-socioeconomicos-en-mexicosegun-la-amai/

INEGI (2020). Resultados de la encuesta nacional de ocupación y empleo. Nueva edición (ENOEN) Cifras durante el tercer trimestre de 2020, Tabasco. https:// www.inegi.org.mx/contenidos / saladeprensa/boletines/2020/enoe_ie/ enoe_ie2020_11_Tab.docx

Kotler, P. y Armstrong, G. (2008). Fundamentos de Marketing 8va edición. Pearson Education, 
México. https://www.academia.edu/42228045/ Kotler_P_and_Armstrong_G_2008_Fundame ntos_de_marketing_8va_edici\%C3\%B3n_Pear son_Educaci\%C3\%B3n_M\%C3\%A9xico

Madrigal, F., Madrigal-Moreno, S., y ÁvilaCarreón, F. (2019). Retos y Oportunidades del Comportamiento Organizacional de los Millennials Como Fuerza de Trabajo. Revista de la facultad de contaduría y ciencias administrativas, 4(7), 86-95. https://rfcca.umich.mx/ index.php/rfcca/article/view/109

Moscoso, V (5-7 de diciembre de 2013). El papel de los actores territoriales en la definición y configuración de modelos de desarrollo turístico. Congreso de Turismo: "El Turismo y los Nuevos Paradigmas Educativos". Consejo de Decanos y Directores de Unidades Académicas relacionadas con la Enseñanza del Turismo y Universidad Nacional de Tierra del Fuego, Antártida e Islas del Atlántico Sur. Ushuaia, Argentina. http:// sedici.unlp.edu.ar/handle/10915/34681

Peñalosa, Mónica y López, Diana (2016). La generación de los millennials frente al consumo socialmente responsable. Cuadernos Latinoamericanos de Administración, vol. XII, núm. 23, 73-81. https://www.redalyc.org/ pdf/4096/409650120008.pdf

Pere-Oriol, C., Pérez, J. M., y Tropea, F. (1996). Tribus urbanas. El ansia de identidad juvenil: entre el culto a la imagen y la autoafirmación a través de la violencia. Barcelona, Paidós Estado y Sociedad.
Ponce, M.J., Besanilla, T. y Rodríguez, H.A. (2012). Factores que influyen en el comportamiento del consumidor. Contribuciones a la Economía. https:// www.eumed.net/ce/2012/dhi.html

Possebon, J., Cervi, C., \& Knebel, D. (2019). Factores que influyen en la decisión de compra de viajes turísticos. Un estudio en el Noroeste de Rio Grande do Sul (Brasil). Estudios y Perspectivas en Turismo, 28(4),903-922. https://www.redalyc.org/ articulo.oa?id=180762638003

Posso, A.M. y Cardona, D. C. (2016). Tendencias de consumo turístico de los Millenials en la ciudad de Ibarra. Revista Ecos de la Academia, Vol 2, Num 4, 145-157. http:// revistasojs.utn.edu.ec/index.php/ ecosacademia/article/view/170/166

Quevedo, I. y Pérez, E. (2018). Generación millennial: consumo gastronómico $y$ turístico. Hitos de ciencias económico administrativas, num 68. https:// revistas.ujat.mx/index.php/hitos/article/ view/2522/1928

Rodríguez, R. (2011). Destinos turísticos. Realidad y concepto. Revista de investigación en turismo y desarrollo local, vol 4, No.11. https://www.eumed.net/rev/turydes/11/ rrf.html

Roselló, V. (S/F). Hábitos turísticos: ¿Cómo viajan los turistas? Hundredrooms y ESERP Business School. https://es.eserp.com/wpcontent/uploads/2017/01/InformeMillenials-HR-ESERP.PDF 
Ruiz Muñoz, W. S., \& Vicente Rodríguez, S. F. (2018). Factores del Comportamiento de Compra y Estrategias de Marketing Mix en los Consumidores de Restaurantes de Comida Saludable de Trujillo - 2018 [Tesis Licenciatura, Universidad César Vallejo]. https://repositorio.ucv.edu.pe/ handle/20.500.12692/26504

Saeteros, A. M., Da Silva, E. V., \& Flores, M. A. (2019). Turismo Sustentable y los diferentes enfoques, aproximaciones $\mathrm{y}$ herramientas para su medición. PASOS Revista De Turismo Y Patrimonio Cultural, 17(5), 901-914. https://doi.org/ 10.25145/j.pasos.2019.17.064

Sánchez, G. (05 de julio de 2017). ¿Quiénes son los MUPPIES y qué efecto tienen en el mercado? Insights. https://www.insights.la/ 2018/07/05/muppies-efecto-en-el-mercado/

Servicio Nacional de Turismo - SERNATUR (2016). MANUAL DE DESTINOS Elementos para la gestión de Destinos Turísticos. Gobierno de Chile. Santiago de Chile. https://www.sernatur.cl/wpcontent/uploads/2018/11/Manual-deDestinos-Elementos-para-lagestio \% C C \% $81 \mathrm{n}$-de-destinosturisticos-1.pdf

Silveira, J. y Silveira, C. (2009). La evolución del marketing de destinos su sinergia con la planificación turística. Estudios $\mathrm{y}$ Perspectivas en Turismo, vol. 18, 530-545. https://www.redalyc.org/pdf/ 1807/180713902002.pdf

Schiffman, L., \& Kanuk, L. (2010). Comportamiento del consumidor.
Pearson Educacion, México. https:// uachatec.com.mx/wp-content/uploads/ $2019 / 10 /$ Comportamiento-delConsumidor-10ed-Schiffman-y-LazarKanuk.pdf

Vidal Fócil, Ana Bertha (2017). El festival del chocolate como estrategia de promoción del turismo cultural en el estado de Tabasco, México. International Journal of Scientific Management and Tourism, Vol 3, No. 2, 501-512. https://dialnet.unirioja.es/descarga/ articulo/6132934.pdf 